\title{
Attenuation correction for hybrid MR/PET scanners: a comparison study
}

\author{
Elena Rota Kops ${ }^{*}$, Andre Santos Ribeiro², Liliana Caldeira ${ }^{1}$, Hubertus Hautzel ${ }^{3}$, Mathias Lukas ${ }^{4}$, Gerald Antoch ${ }^{3}$, \\ Christoph Lerche ${ }^{1}$, Jon Shah ${ }^{1}$ \\ From PSMR 2015: 4th Conference on PET/MR and SPECT/MR \\ La Biodola, Isola d'Elba, Italy. 17-21 May 2015
} ${ }^{1}$ Forschungszentrum Jülich $\mathrm{GmbH}$,
Jülich, Germany
Attenuation correction of PET data acquired in hybrid MR/PET scanners is still a challenge. Different methods have been adopted by several groups to obtain reliable attenuation maps (mu-maps). In this study we compare three methods: MGH, UCL, Neural-Network. The MGH method is based on an MR/CT template obtained with the SPM8 software. The UCL method uses a database of MR/CT pairs. Both generate mu-maps from MP-RAGE images. The feed-forward neural-network from Juelich (NN-Juelich) requires two UTE images; it generates segmented mu-maps. Data from eight subjects (S1-S8) measured in the Siemens 3T MR-BrainPET scanner were used. Corresponding $\mathrm{CT}$ images were acquired. The resulting mu-maps were compared against the CT-based mu-maps for each subject and method. Overlapped voxels and Dice similarity coefficients, D, for bone, soft-tissue and air regions, and relative differences images were calculated. The true positive (TP) recognized voxels for the whole head were $79.9 \%$ (NN-Juelich, S7) to $92.1 \%$ (UCL method, S1). D values of the bone were $\mathrm{D}=0.65$ (NN-Juelich, S1) to $\mathrm{D}=0.87$ (UCL method, S1). For S8 the MHG method failed ( $\mathrm{TP}=76.4 \%$; $\mathrm{D}=0.46$ for bone). $\mathrm{D}$ values shared a common tendency in all subjects and methods to recognize soft-tissue as bone. The relative difference images showed a variation of $-10.9 \%-+10.1 \%$; for S8 and MHG method the values were $-24.5 \%$ and $+14.2 \%$. A preliminary comparison of three methods for generation of mumaps for MR/PET scanners is presented. The continuous methods (MGH, UCL) seem to generate reliable mu-maps, whilst the binary method seems to need further improvement. Future work will include more subjects, the reconstruction of corresponding PET data and their comparison.

\section{Authors' details}

${ }^{1}$ Forschungszentrum Jülich GmbH, Jülich, Germany. ${ }^{2}$ Imperial College London, London, UK. ${ }^{3}$ Heinrich-Heine-University Düsseldorf, Düsseldorf, Germany. ${ }^{4}$ Technische Universitaet Muenchen, Munich, Germany.

Published: 18 May 2015

doi:10.1186/2197-7364-2-S1-A38

Cite this article as: Rota Kops et al: Attenuation correction for hybrid MR/PET scanners: a comparison study. EJNMMI Physics 2015 2(Suppl 1):A38.

\section{SpringerOpen ${ }^{\circ}$}

(C) 2015 Rota Kops et al; licensee Springer. This is an Open Access article distributed under the terms of the Creative Commons Attribution License (http://creativecommons.org/licenses/by/4.0), which permits unrestricted use, distribution, and reproduction in any medium, provided the original work is properly cited. 